\title{
A unified strategy for the synthesis of amorfrutins $A$ and $B$ and evaluation of their cytotoxicity
}

\author{
Benjamin Brandes ${ }^{1}$, Sophie Hoenke ${ }^{1}$, Michael Türk ${ }^{1}$, Björn Weber ${ }^{1}$, Hans-Peter Deigner ${ }^{2}$, Ahmed Al- \\ Harrasi ${ }^{3}$ and René Csuk ${ }^{1, *}$ \\ ${ }^{1}$ Full Address: Martin-Luther-University Halle-Wittenberg, Organic Chemistry, Kurt-Mothes-Str. 2, D-06120 \\ Halle (Saale), Germany \\ ${ }^{2}$ Full Address: Furtwangen University, Institute of Precision Medicine, Medical and Life Sciences Faculty, \\ Jakob-Kienzle-Straße 17, D-78054 Villingen-Schwenningen, Germany \\ ${ }^{4}$ Full Address: University of Nizwa, Chair of Oman's Medicinal Plants and Marine Natural Products, P.O. Box \\ 33, PC 616, Birkat Al-Mauz, Nizwa, Sultanate of Oman
}

\begin{abstract}
Dimethoxy-benzaldehyde was used as a starting material to synthesize a central intermediate, 2hydroxy-4-methoxy-6-phenethylbenzoic acid that was converted very quickly and with good yields into amorfrutins A and B. Furthermore, this compound was also used as a starting material to synthesize a piperazinylrhodamine $\mathrm{B}$ conjugate. The latter compound showed good cytotoxicity $\left(\mathrm{EC}_{50}=2.3-5.1 \mu \mathrm{M}\right)$ and promising selective cytotoxicity $(\mathrm{S}=2.1-4.6$ ) for human tumor cell lines as compared to non-malignant fibroblasts (NIH 3T3).
\end{abstract}

Keywords: Amorfrutin A; Amorfrutin B; synthesis; cytotoxicity.

\section{Introduction}

The vital role of secondary natural products for the development of new drugs is undisputed ${ }^{1-3}$. For thousands of years, people have been using the almost inexhaustible reservoir of plant ingredients ${ }^{4}$ of the socalled "God's pharmacy" 5 . For example, in infectious diseases and cancer 75 and $60 \%$ of new drugs originate from natural sources ${ }^{1-3}$. The global market for pharmaceuticals is about 1.1 trillion US\$; thereby, $35 \%$ of the medicines have developed from natural products ${ }^{1-3}$. Cancer, infectious diseases, and complex non-communicable diseases are still the most frequent causes of death worldwide ${ }^{6}$. Of particular interest are phenolic compounds; they are widely dispersed throughout the plant kingdom, and more than 10.000 different phenolic structures have been isolated so far. For many of them, cytotoxic or anticancer activity has been reported. But also for cardiovascular diseases, which are often also associated with type II diabetes mellitus and obesity, there is an unsustainable burden on society ${ }^{7}$. A new strategy for early invention and prevention consists of the timely application of antidiabetic and lipid-lowering compounds such as the 2-hydroxybenzoic acid-derived amorfrutins ${ }^{8-10}$.

Amorfrutins A (1) and B (2) (Fig. 1) were initially isolated from parts of the bastard indigo-bush Amorpha fruticosa ${ }^{11,12}$. Still, these and other

*Corresponding author: René Csuk

Email address: rene.csuk@chemie.uni-halle.de

DOI: http://dx.doi.org/10.13171/mjc10902011171546rc amorfrutins have also been found in other plants, such as the licorice species Glycyrrhiza foetida ${ }^{13-16}$. A. fruticosa is an indigenous American shrub, while G. foetida is a photoautotrophic plant in the family of Fabaceae. The physiological effects of amorfrutins can be attributed, in part, to selective activation of the nuclear receptor peroxisome proliferator-activated receptor gamma (PPAR $\gamma)^{14,17-21}$. PPAR $\gamma$ regulates genes of glucose and fatty acid metabolism. However, the complex also appears to be important in treating cancer ${ }^{12,16,22}$, inflammations ${ }^{23,24}$, and for impending the age-related decline of metabolism ${ }^{13,15,25-27}$.

Several syntheses have been published to access amorfrutins A and B whereby the former has been the focus of scientific interest. In contrast, the number of syntheses for the latter has remained small 28-34. However, of particular interest are synthetic strategies that allow in principle to synthesize as many as possible of the previously known amorfrutins and, if necessary, analogs in a unified manner ${ }^{31,33}$.

A most recently published synthesis of amorfrutin B from amorfrutin A methyl ester seems particularly worth mentioning since it holds a critical step in a Johnson-Claisen rearrangement reaction 33,35 . However, this method's elegance is diminished by the available length of the synthesis and the sometimes only moderate yields.

Received September 16, 2020

Accepted November 5, 2020

Published January 3, 2020 
<smiles>COc1cc(CCc2ccccc2)c(C(=O)O)c(O)c1CC=C(C)C</smiles>

Amorfrutin A (1)<smiles>COc1cc(CCc2ccccc2)c(C(=O)O)c(O)c1C/C=C(\C)CCC=C(C)C</smiles>

Amorfrutin B (2)

Figure 1. Structure of amorfrutins A (1) and B (2) highlighting the 2-hydroxybenzoic acid core structure

\section{Results and discussion}

In the course of our syntheses, we could show that compound $\mathbf{3}$ (Scheme 1) is easily accessible in large quantities from commercial 3,5-dimethoxybenzaldehyde in only 6 steps in a total yield of $63 \%{ }^{34}$. Thus, 3 seems to be an ideal starting material for synthesizing the two amorfrutins $\mathrm{A}$ and $\mathrm{B}$.

Regarding the synthesis of amorfrutins, $\mathbf{3}$ was converted into methyl ester $\mathbf{4}$ by reaction with $\mathrm{MeI}$ in the presence of $\mathrm{Cs}_{2} \mathrm{CO}_{3}$ in $98 \%$ yield. The reaction of $4^{34,36}$ with K.H. and prenyl chloride gave a mixture of
5 (as a product of C-alkylation) ${ }^{34,37}$, and $\mathbf{6}$ (as an etherification product) ${ }^{13}$. Whereas, from the reaction with geranyl chloride, a mixture of 7 (from a C-alkylation) ${ }^{17}$ and 8 (C- and O-alkylation) was obtained. Both mixes were easily separated by column chromatography. Hydrolysis of 5 furnished amorfrutin A (1) while from the hydrolysis of 7, amorfrutin B (2) was obtained in $84 \%$ isolated yield. The side products of the former reactions, $\mathbf{6}$ and $\mathbf{8}$ were transformed by their reaction with $\mathrm{CeCl}_{3}$ in acetonitrile in the presence of $\mathrm{NaI}$ very easily into starting material $\mathbf{4}$ and amorfrutin B 2, respectively.

Table 1. Cytotoxicity of compounds 1-10 and rhodamine B (Rho) a

\begin{tabular}{|c|c|c|c|c|c|c|}
\hline Compound & A375 & HT29 & MCF-7 & A2780 & $\mathrm{FaDu}$ & NIH $3 \mathrm{~T} 3$ \\
\hline Rho & $>30$ & $>30$ & $>30$ & $>30$ & $>30$ & $>30$ \\
\hline 1 & $>30$ & $>30$ & $>30$ & $>30$ & $>30$ & $>30$ \\
\hline 2 & $23.8 \pm 2.0$ & $>30$ & $26.2 \pm 1.4$ & $25.0 \pm 2.0$ & $27.1 \pm 0.9$ & $>30$ \\
\hline 3-5 & $>30$ & $>30$ & $>30$ & $>30$ & $>30$ & $>30$ \\
\hline 6 & $>30$ & $>30$ & $>30$ & $22.9 \pm 1.8$ & $>30$ & $>30$ \\
\hline 7 & $>30$ & $>30$ & $28.9 \pm 3.8$ & $20.5 \pm 1.9$ & $>30$ & $>30$ \\
\hline 8 & $19.6 \pm 2.3$ & $>30$ & $12.0 \pm 1.2$ & $19.8 \pm 2.1$ & $22.0 \pm 2.3$ & $>30$ \\
\hline 9 & $>30$ & $>30$ & $17.8 \pm 3.9$ & $26.4 \pm 2.1$ & $>30$ & $>30$ \\
\hline 10 & $\begin{array}{c}4.7 \pm 0.3 \\
0\end{array}$ & $5.1 \pm 0.2$ & $\begin{array}{l}3.4 \pm 0.5 \\
0\end{array}$ & $2.3 \pm 0.3$ & $3.7 \pm 0.2$ & $1.1 \pm 0.1$ \\
\hline STA & $0.2 \pm 0.02$ & $0.1 \pm 0.01$ & $0.1 \pm 0.01$ & $0.1 \pm 0.05$ & $0.008 \pm 0.001$ & $0.2 \pm 0.02$ \\
\hline
\end{tabular}

a $\left(\mathrm{EC}_{50}\right.$ values in $\mu \mathrm{M}$ from SRB assays after $72 \mathrm{~h}$ of treatment, the values are averaged from three independent experiments performed each in triplicate, confidence interval CI $=95 \%$; mean \pm standard mean error, cut-off 30 $\mu \mathrm{M})$. Human cancer cell lines: A375 (epithelial melanoma), HT29 (colorectal adenocarcinoma), MCF-7 (breast adenocarcinoma), A2780 (ovarian carcinoma), FaDu (squamous cell carcinoma); non-malignant: NIH 3T3 (mouse fibroblasts). Staurosporine (STA) was used as a positive standard; 
Relatively little is known about the possible cytotoxicity of amorfrutins A and B. Thus, these two compounds were investigated in an SRB assay. This showed amorfrutin A (1, Table 1) not to be cytotoxic for several human tumor cell lines as well as for nonmalignant mouse fibroblasts (NIH 3T3). EC 50 values between $23.8 \mu \mathrm{M}$ (for A375 cells) and $27.1 \mu \mathrm{M}$ (for $\mathrm{FaDu}$ cells), however, were observed for amorfrutin B (2).<smiles>COc1cc(C=O)cc(OC)c1</smiles><smiles>COc1cc(O)c(C(=O)O)c(CCc2ccccc2)c1</smiles><smiles>COc1cc(CCc2ccccc2)c(C(=O)Cl)c(O)c1CC=C(C)C</smiles>

3<smiles>COc1cc(O)c(C(=O)O)c(CCc2ccccc2)c1</smiles><smiles>COc1cc(O)c(C(=O)O)c(CCc2ccccc2)c1</smiles><smiles>[R]OC(=O)c1c(O)cc(OC)cc1CCC(=C)C</smiles><smiles>COc1ccc(C)c(OCC=C(C)C)c1</smiles>

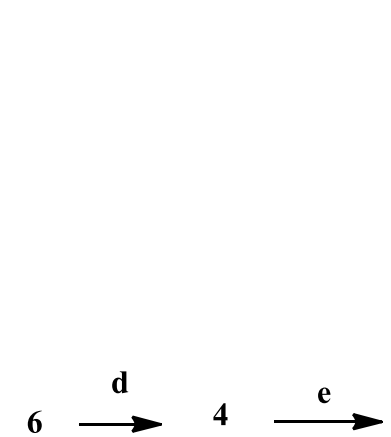<smiles>C=C(/C=C\CCC1=CC=CCC1)OC</smiles><smiles>CC=C(C)CCC=C(C)C</smiles><smiles>[18F]</smiles> 
Hybrid compound $\mathbf{1 0}$ held good cytotoxicity (Table 1) in low micro-molar concentration for all human tumor cell lines, combined with promising selectivity.<smiles>CCN(CC)c1ccc2c(-c3ccccc3C(=O)[O-])c3ccc(=[N+](CC)CC)cc-3oc2c1</smiles>

Rhodamine B<smiles>CCN(CC)c1ccc2c(-c3ccccc3C(=O)N3CCNCC3)c3ccc(=[N+](CC)CC)cc-3oc2c1</smiles>

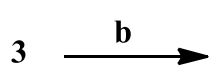<smiles>CCN(CC)c1ccc2c(-c3ccccc3C(=O)N3CCN(C(=O)c4c(O)cc(OC)cc4CCc4ccccc4)CC3)c3ccc(=[N+]([O-])CC)cc-3oc2c1</smiles>

Scheme 2. Synthesis of compounds 9 and 10: reactions and conditions: a) DCM, $(\mathrm{COCl})_{2}$, DMF, piperazine, $24 \mathrm{~h}, 25^{\circ} \mathrm{C}, 67 \%$; b) EDC x HCl, HOBt, DCM, 9, 12 h, $25^{\circ} \mathrm{C}, 74 \%$

\section{Conclusion}

From 3,5-dimethoxy-benzaldehyde, a central intermediate, 2-hydroxy-4-methoxy-6-phenethylbenzoic acid is accessible in only 6 steps, which can be converted very easily and with good yields into the amorfrutins A and B. This intermediate, however, can also serve as a starting material for the synthesis of amides. In this case, piperazinyl-rhodamine B conjugate showed good cytotoxicity for several human tumor cell lines (A375, HT29, MCF-7, A2780, $\mathrm{FaDu})$ as well as promising tumor/non-tumor cell selectivity.

\section{Acknowledgments}

The authors are grateful to Dr. D. Ströhl, Y. Schiller, and S. Ludwig for multiple NMR spectra and to the late Dr. R. Kluge for M.S. measurements. Th. Schmidt recorded additional M.S. spectra. M. Schneider and S. Ludwig performed I.R. and U.V./Vis spectra as well as elemental analyses. The cell lines were provided by Dr. T. Müller (Dept. of Oncology).

\section{Experimental}

Instrumentation was previously described ${ }^{38-46}$. Starting materials were obtained from local suppliers in bulk, and the solvents (technical grade) were redistilled and dried according to usual procedures. All reactions were performed under argon using ovendried glassware. The routine aqueous workup included the dilution of the reaction mixture with the solvent (used for the reaction), aqueous extraction, reextraction of the aqueous phase (twice), drying of the combined organic phases $\left(\mathrm{MgSO}_{4}\right)$, and evaporation of the organic phase under reduced pressure.

\subsection{Cytotoxic evaluation}

The cell lines were obtained from the Department of Oncology (Martin-Luther-University Halle Wittenberg). Cultures were maintained as monolayers in RPMI 1640 medium with L-glutamine (Capricorn Scientific GmbH, Ebsdorfergrund, Germany) supplemented with $10 \%$ heat-inactivated fetal bovine serum (Sigma-Aldrich Chemie GmbH, Steinheim, Germany) and penicillin/streptomycin (Capricorn Scientific GmbH, Ebsdorfergrund, Germany) at $37^{\circ} \mathrm{C}$ in a humidified atmosphere with $5 \% \quad \mathrm{CO}_{2}$. The compounds' cytotoxicity was evaluated using the sulforhodamine-B (Kiton-Red S, ABCR) micro- 
culture colorimetric assay, as previously reported ${ }^{38-46}$. In short, the cells were seeded into 96 well plates on day zero at appropriate cell densities to prevent the confluence of the cells during the experiment. After 24 hours, the cells were treated with different concentrations $(1,3,7,12,20$, and $30 \mu \mathrm{M})$, but the final concentration of DMSO/DMF never exceeded $0.5 \%$, which was non-toxic to the cells. After $72 \mathrm{~h}$ treatment, the supernatant media from the 96 well plates were discarded, then the cells were fixed with $10 \%$ trichloroacetic acid and allowed to rest at $4^{\circ} \mathrm{C}$. After 24 hours of fixation, the cells were washed in a strip washer and then dyed with SRB solution $(200 \mu \mathrm{L}, 10 \mathrm{mM})$ for 20 minutes. The plates were then washed four times with $1 \%$ acetic acid to remove the dye's excess and allowed to air-dry overnight. Tris base solution $(200 \mu \mathrm{L}, 10 \mathrm{mM})$ was added to each well. The absorbance was measured with a 96 well plate reader from Tecan Spectra.

\section{Methyl 2-hydroxy-4-methoxy-6- phenethylbenzoate (4)}

From 3: A suspension of $\mathbf{3}(2.00 \mathrm{~g}, 7.34 \mathrm{mmol})$ and $\mathrm{Cs}_{2} \mathrm{CO}_{3}(4.8 \mathrm{~g}, 7.8 \mathrm{mmol})$ in dry DMF $(40 \mathrm{~mL})$ was stirred for $10 \mathrm{~min}$; iodomethane $(0.72 \mathrm{~mL}, 10.8 \mathrm{mmol})$ was added, and the stirring was continued for $12 \mathrm{~h}$. Usual workup gave $4(2.02 \mathrm{~g}, 96 \%)$ as a colorless oil pure enough for the transformations to follow.

From 6: To a solution of $6(150 \mathrm{mg}, 0.42 \mathrm{mmol})$ in acetonitrile $(5 \mathrm{~mL}) \quad \mathrm{CeCl}_{3} \times 7 \quad \mathrm{H}_{2} \mathrm{O} \quad(190 \quad \mathrm{mg}$, $0.51 \mathrm{mmol})$ and $\mathrm{NaI}(80 \mathrm{mg}, 0.53 \mathrm{mmol})$ were added. The mixture was stirred at $25^{\circ} \mathrm{C}$ for $12 \mathrm{~h}$. Usual aqueous work-up followed by chromatography (silica gel, $n$-hexane/ethyl acetate, 6:1) gave 4 (115 mg, $95 \%$ ); m.p. $49-51{ }^{\circ} \mathrm{C}$ (lit.: ${ }^{34} 50-52^{\circ} \mathrm{C}$ ); $\mathrm{R}_{\mathrm{F}}=0.45$ (n-hexane/ethyl acetate, 12:1);

IR (ATR): $\tilde{\mathrm{V}}=2952 \mathrm{w}, 1650 \mathrm{~m}, 1614 \mathrm{~m}, 1575 \mathrm{~m}$, $1496 \mathrm{w}, 1434 \mathrm{~m}, 1380 \mathrm{w}, 1325 \mathrm{~s}, 1203 \mathrm{~m}, 1253 \mathrm{~s}, 1203 \mathrm{~s}$, $1156 \mathrm{~s}, 1110 \mathrm{~m}, 1047 \mathrm{~m}, 956 \mathrm{~m}, 802 \mathrm{~m}, 748 \mathrm{~m}, 698 \mathrm{~s}$, 638w, 599 $\mathrm{w} \mathrm{cm}^{-1}$;

UV/Vis $\left(\mathrm{CHCl}_{3}\right): \lambda_{\max }(\log \varepsilon)=230$ (4.30), 265 (4.19), 304 (3.82) nm;

${ }^{1} \mathrm{H}$ NMR $\left(500 \mathrm{MHz}, \mathrm{CDCl}_{3}\right) \delta=11.75(\mathrm{~s}, 1 \mathrm{H}, \mathrm{OH})$, $7.34-7.16(\mathrm{~m}, 5 \mathrm{H}, 10-\mathrm{H}, 11-\mathrm{H}, 12-\mathrm{H}), 6.38(\mathrm{~d}, \mathrm{~J}=$ $2.6 \mathrm{~Hz}, 1 \mathrm{H}, 5-\mathrm{H}), 6.29$ (d, J = 2.6 Hz, 1H, 3-H), 3.96 (s, 3H, COOMe), 3.79 (s, 3H, OMe), $3.21-3.14$ $\left(\mathrm{m}, 2 \mathrm{H}, 7-\mathrm{H}_{2}\right), 2.88-2.82\left(\mathrm{~m}, 2 \mathrm{H}, 8-\mathrm{H}_{2}\right) \mathrm{ppm}$;

${ }^{13} \mathrm{C} \mathrm{NMR}\left(126 \mathrm{MHz}, \mathrm{CDCl}_{3}\right): \delta=171.9(\mathrm{COOH})$, 165.8 (C-4), 164.2 (C-2), 146.8 (C-6), 142.1 (C-9), 128.6 (C-10), 128.5 (C-11), 126.1 (C-12), 111.0 (C-5), 104.8 (C-1), 99.4 (C-3), 55.4 (OMe), 52.2 (COOMe), 39.0 (C-7), 38.4 (C-8) ppm;

MS (ESI, MeOH): $\mathrm{m} / \mathrm{z}=287.1\left(100,[\mathrm{M}+\mathrm{H}]^{+}\right)$; analysis calcd for $\mathrm{C}_{17} \mathrm{H}_{18} \mathrm{O}_{4}$ (286.32): C 71.31, H 6.34; found: C 71.11, H 6.60.

Methyl 2-hydroxy-4-methoxy-3-(3-methyl-2buten-1-yl)-6-phenylethylbenzoate (5) and methyl 4-methoxy-2-[(3-methylbut-2-en-1-yl)oxy]-6phenethylbenzoate (6)
A solution of $4(1.0 \mathrm{~g}, 3.49 \mathrm{mmol})$ in dry toluene

(30 mL) and K.H. (154 mg, $3.84 \mathrm{mmol}$; K.H. was obtained as a suspension in mineral oil. Before the reaction, the suspension was washed in a Schlenk-frit with dry $n$-hexane to remove the oil. The pure K.H. was dried in a stream of dry argon) was stirred at $25^{\circ} \mathrm{C}$ for $20 \mathrm{~min}$, followed by $20 \mathrm{~min}$ at $70^{\circ} \mathrm{C}$. At $25^{\circ} \mathrm{C}$, prenyl chloride (440 mg, $4.21 \mathrm{mmol}$ ) was added, and the mixture was stirred at $75^{\circ} \mathrm{C}$ for 2 hours. Usual workup followed by chromatography (silica gel, $n$-hexane/ethyl acetate, 12:1) gave $5(890 \mathrm{mg}, 72 \%)$ and 6 (301 $\mathrm{mg}, 24 \%)$.

Data for 5: colorless solid; m.p. $67-69^{\circ} \mathrm{C}$ (lit.: ${ }^{34}$ m.p. 67.9 C); $\mathrm{R}_{\mathrm{F}}=0.58$ ( $n$-hexane/ethyl acetate, 12:1); IR (ATR): $\tilde{\mathrm{V}}=2924 \mathrm{~m}, 1655 \mathrm{~m}, 1603 \mathrm{~m}, 1573 \mathrm{~m}$, $1494 \mathrm{w}, 1435 \mathrm{~m}, 1405 \mathrm{~m}, 1288 \mathrm{~s}, 1224 \mathrm{~s}, 1154 \mathrm{~s}, 1112 \mathrm{~s}$, $1004 \mathrm{~m}, 962 \mathrm{w}, 804 \mathrm{~s}, 773 \mathrm{~m}, 737 \mathrm{~s}, 700 \mathrm{~s}, 656 \mathrm{~m}, 616 \mathrm{w}$, $558 \mathrm{w}, 522 \mathrm{~m} \mathrm{~cm}^{-1}$;

UV/Vis $(\mathrm{MeOH}): \lambda_{\max }(\log \varepsilon)=226(4.37), 270$ (3.99), 308 (3.55) nm;

${ }^{1} \mathrm{H}$ NMR $\left(500 \mathrm{MHz}, \mathrm{CDCl}_{3}\right) \delta=11.72(\mathrm{~s}, 1 \mathrm{H}, \mathrm{OH})$, $7.33-7.17$ (m, 5H, 10-H, 11-H, 12-H), $6.21(\mathrm{~s}, 1 \mathrm{H}$, $5-\mathrm{H}), 5.23-5.17(\mathrm{~m}, 1 \mathrm{H}, 14-\mathrm{H}), 3.96(\mathrm{~s}, 3 \mathrm{H}$, COOMe), 3.80 (s, 3H, OMe), 3.34 (d, J = 7.0 Hz, 2H, $\left.13-\mathrm{H}_{2}\right), 3.21-3.14\left(\mathrm{~m}, 2 \mathrm{H}, 7-\mathrm{H}_{2}\right), 2.88-2.82(\mathrm{~m}, 2 \mathrm{H}$, 8- $\left.\mathrm{H}_{2}\right), 1.78$ (s, 3H, $\left.\mathrm{CH}_{3}\right), 1.68\left(\mathrm{~s}, 3 \mathrm{H}, \mathrm{CH}_{3}\right) \mathrm{ppm}$;

${ }^{13} \mathrm{C} \mathrm{NMR}\left(126 \mathrm{MHz}, \mathrm{CDCl}_{3}\right): \delta=172.3(\mathrm{COOH})$, 162.0 (C-4), 161.4 (C-2), 144.2 (C-6), 142.2 (C-9), 131.8 (C-15), 128.5 (C-10, C-11), 126.1 (C-12), 122.5 (C-14), 115.4 (C-3), 106.1 (C-5), 105.3 (C-1), 55.6 (OMe), 52.1 (COOMe), 39.4 (C-7), 38.6 (C-8), 25.9 $\left.\left(\mathrm{CH}_{3}\right), 22.1(\mathrm{C}-13)\right), 17.9\left(\mathrm{CH}_{3}\right) \mathrm{ppm}$;

MS (ESI, MeOH): $\mathrm{m} / \mathrm{z}=355.1\left(15,[\mathrm{M}+\mathrm{H}]^{+}\right), 377.2$ $\left(100,[\mathrm{M}+\mathrm{Na}]^{+}\right)$;

analysis calcd for $\mathrm{C}_{22} \mathrm{H}_{26} \mathrm{O}_{4}$ (354.5): C 74.55, H 7.39; found: C 74.32, H 7.54.

Data for 6: pale yellowish oil; $\mathrm{R}_{\mathrm{F}}=0.45$ (n-hexane/ethyl acetate, 12:1);

IR (ATR): $\tilde{\mathrm{V}}=2963 \mathrm{w}, 1602 \mathrm{~m}, 1495 \mathrm{w}, 1435 \mathrm{~m}$, $1364 \mathrm{~m}, 1259 \mathrm{~s}, 1218 \mathrm{~m}, 1204 \mathrm{~m}, 1159 \mathrm{~s}, 1103 \mathrm{~s}, 1032 \mathrm{~m}$, $955 \mathrm{w}, 883 \mathrm{w}, 850 \mathrm{w}, 797 \mathrm{~s}, 748 \mathrm{~m}, 725 \mathrm{w}, 697 \mathrm{~s}$, $604 \mathrm{~m} \mathrm{~cm}^{-1}$;

$\mathrm{UV} / \mathrm{Vis}\left(\mathrm{CHCl}_{3}\right): \lambda_{\max }(\log \varepsilon)=229(4.25) \mathrm{nm}$;

${ }^{1} \mathrm{H}$ NMR $\left(400 \mathrm{MHz}, \mathrm{CDCl}_{3}\right) \delta=7.33-7.15(\mathrm{~m}, 5 \mathrm{H}$, $10-\mathrm{H}, 11-\mathrm{H}, 12-\mathrm{H}), 6.34$ (d, J = $2.2 \mathrm{~Hz}, 1 \mathrm{H}, 5-\mathrm{H}), 6.23$ $(\mathrm{d}, \mathrm{J}=2.2 \mathrm{~Hz}, 1 \mathrm{H}, 3-\mathrm{H}), 5.43(\mathrm{t}, J=6.3 \mathrm{~Hz}, 1 \mathrm{H}$, $14-\mathrm{H}), 4.53\left(\mathrm{~d}, \mathrm{~J}=6.5 \mathrm{~Hz}, 2 \mathrm{H}, 13-\mathrm{H}_{2}\right), 3.89(\mathrm{~s}, 3 \mathrm{H}$, COOMe), 3.75 (s, 3H, OMe), $2.94-2.80(\mathrm{~m}, 4 \mathrm{H}$, 7- $\left.\mathrm{H}_{2}, 8-\mathrm{H}_{2}\right), 1.77$ (s, 3H, $\left.\mathrm{CH}_{3}\right), 1.72\left(\mathrm{~s}, 3 \mathrm{H}, \mathrm{CH}_{3}\right) \mathrm{ppm}$; ${ }^{13} \mathrm{C}$ NMR (101 MHz, $\left.\mathrm{CDCl}_{3}\right): \delta=168.9(\mathrm{COOH})$, 161.4 (C-4), 157.6 (C-2), 141.9 (C-6), 141.8 (C-9), 137.5 (C-15), 128.6 (C-10), 128.5 (C-11), 126.1 (C-12), 119.9 (C-14), 117.0 (C-1), 106.2 (C-5), 98.1 (C-3), 66.1 (C-13)), 55.5 (OMe), 52.2 (COOMe), 37.8 (C-7), 36.4 (C-8), $25.7\left(\mathrm{CH}_{3}\right), 18.4\left(\mathrm{CH}_{3}\right) \mathrm{ppm}$; MS $(\mathrm{ESI}, \mathrm{MeOH}): \mathrm{m} / \mathrm{z}=355.0\left(20,[\mathrm{M}+\mathrm{H}]^{+}\right), 377.1$ $\left(100,[\mathrm{M}+\mathrm{Na}]^{+}\right)$; analysis calcd for $\mathrm{C}_{22} \mathrm{H}_{26} \mathrm{O}_{4}$ (354.5): C 74.55, H 7.39; found: C 74.31, H 7.58. 
Amorfrutin A, 2-hydroxy-4-methoxy-3-(3methylbut-2-en-1-yl)-6-phenethylbenzoic acid (1) To a solution of $\mathrm{KOH}(760 \mathrm{mg}, 13.56 \mathrm{mmol})$ in $\mathrm{MeOH} / \mathrm{H}_{2} \mathrm{O}(7: 1,14 \mathrm{~mL})$ a solution of $5(800 \mathrm{mg}$, $2.26 \mathrm{mmol})$ in $\mathrm{MeOH}(20 \mathrm{~mL})$ was added. After heating under reflux for $8 \mathrm{~h}$ followed by usual aqueous work-up and chromatography (silica gel, n-hexane/ethyl acetate, 4:1) amorfrutin A (1, $696 \mathrm{mg}$, $91 \%$ ) was obtained as an off-white solid; m.p. $111-113^{\circ} \mathrm{C}$ (lit.: ${ }^{34}$ m.p. $113.7^{\circ} \mathrm{C}$ ); $\mathrm{R}_{\mathrm{F}}=0.33$ (n-hexane/ethyl acetate, $4: 1$ );

IR (ATR): $\tilde{\mathrm{v}}=2925 \mathrm{w}, 1610 \mathrm{~s}, 1495 \mathrm{w}, 1453 \mathrm{~m}, 1435 \mathrm{w}$, $1267 \mathrm{~s}, 1228 \mathrm{~s}, 1175 \mathrm{~s}, 1115 \mathrm{~s}, 1040 \mathrm{~m} \mathrm{~cm}^{-1}$;

$\mathrm{UV} / \mathrm{Vis}(\mathrm{MeOH}): \lambda_{\max }(\log \varepsilon)=224(4.35), 265$ (3.83), 305 (3.42) nm;

${ }^{1} \mathrm{H}$ NMR $\left(500 \mathrm{MHz}, \mathrm{CDCl}_{3}\right): \delta=11.90(\mathrm{~s}, 1 \mathrm{H}, \mathrm{OH})$, $7.35-7.15$ (m, 5H, 10-H, 11-H, 12-H), 6.25 (s, 1H, 5-H), $5.26-5.15(\mathrm{~m}, 1 \mathrm{H}, 14-\mathrm{H}), 3.82$ (s, 3H, OMe), $3.38\left(\mathrm{~d}, \mathrm{~J}=7.0 \mathrm{~Hz}, 2 \mathrm{H}, 13-\mathrm{H}_{2}\right), 3.30-3.23(\mathrm{~m}, 2 \mathrm{H}$, 7- $\left.\mathrm{H}_{2}\right), 2.98-2.90\left(\mathrm{~m}, 2 \mathrm{H}, 8-\mathrm{H}_{2}\right), 1.81\left(\mathrm{~s}, 3 \mathrm{H}, \mathrm{CH}_{3}\right)$, $1.71\left(\mathrm{~s}, 3 \mathrm{H}, \mathrm{CH}_{3}\right) \mathrm{ppm}$;

${ }^{13} \mathrm{C}$ NMR $\left(126 \mathrm{MHz}, \mathrm{CDCl}_{3}\right): \delta=177.1(\mathrm{COOH})$, 163.4 (C-4), 162.0 (C-2), 145.7 (C-6), 142.3 (C-9), 131.9 (C-15), 128.5 (C-10), 128.4 (C-11), 126.0 (C12), 122.6 (C-14), 115.7 (C-3), 106.7 (C-5), 103.7 (C1), $55.6(\mathrm{OMe}), 39.6(\mathrm{C}-7), 38.5(\mathrm{C}-8), 25.7\left(\mathrm{CH}_{3}\right)$, $22.0(\mathrm{C}-13), 17.9\left(\mathrm{CH}_{3}\right) \mathrm{ppm}$;

ESI-MS $(\mathrm{MeOH}): \mathrm{m} / \mathrm{z}=341.0\left(50,[\mathrm{M}+\mathrm{H}]^{+}\right), 363.1$ $\left(100,[\mathrm{M}+\mathrm{Na}]^{+}\right)$;

analysis calcd for $\mathrm{C}_{21} \mathrm{H}_{24} \mathrm{O}_{4}$ (340.41): C 74.09; H 7.11; found: $\mathrm{C} 73.91$; H 7.30.

\section{Methyl (E)-3-(3,7-dimethylocta-2,6-dien-1-yl)-2- hydroxy-4-methoxy-6-phenethylbenzoate (7) and methyl 3-((E)-3,7-dimethylocta-2,6-dien-1-yl)-2- (( $(E)-3,7-d i m e t h y l o c t a-2,6-d i e n-1-y l) o x y)-4-$ methoxy-6 phenethylbenzoate $(8)$}

To a suspension of K.H. (110 mg, $2.74 \mathrm{mmol})$ in dry toluene $(100 \mathrm{~mL})$, a solution of $\mathbf{4}(714 \mathrm{mg}, 2.49 \mathrm{mmol})$ in dry toluene $(50 \mathrm{~mL})$ was slowly added, and the mixture was stirred at $23^{\circ} \mathrm{C}$ for $15 \mathrm{~min}$ followed by the addition of geranyl chloride $(560 \mu \mathrm{L}, 3.04 \mathrm{mmol})$. The stirring at $70^{\circ} \mathrm{C}$ was continued for $12 \mathrm{~h}$. Usual aqueous workup followed by chromatography (silica gel, $n$-hexane/ethyl acetate, 12:1) gave 7 (778 mg, $74 \%)$ and 8 (114 $\mathrm{mg}, 8 \%)$ each as a colorless oil.

Data for 7: $\mathrm{R}_{\mathrm{F}}=0.67$ ( $n$-hexane/ethyl acetate, 9:1); IR (ATR): $v=3085 v w, 3061 v w, 3027 w, 2952 w$, $2916 w, 2854 w, 1725 w, 1651 s, 1609 m, 1573 m$, $1496 \mathrm{w}, 1452 \mathrm{~m}, 1436 \mathrm{~m}, 1404 \mathrm{~m}, 1376 \mathrm{~m}, 1280 \mathrm{vs}$, $1226 s, 1194 m, 1155 s, 1112 s, 1077 w, 1043 w$, $1030 w, 1007 m, 962 w, 912 w, 882 w, 836 w, 807 m$, $770 m, 748 m, 699 s, 659 w, 608 m, 557 w, 488 w$, $470 w \mathrm{~cm}^{-1}$;

${ }^{1} \mathrm{H}$ NMR (400 MHz, $\left.\mathrm{CDCl}_{3}\right): \delta=11.73(s, 1 \mathrm{H}, \mathrm{OH})$, $7.34-7.26(m, 2 \mathrm{H}, 11-\mathrm{H}), 7.25-7.17(m, 3 \mathrm{H}, 10-\mathrm{H}$, $12-\mathrm{H}), 6.22(s, 1 \mathrm{H}, 5-\mathrm{H}), 5.22(t q, J=7.0,1.3 \mathrm{~Hz}$, $1 \mathrm{H}, 17-\mathrm{H}), 5.08(t q, J=7.0,1.4 \mathrm{~Hz}, 1 \mathrm{H}, 21-\mathrm{H}), 3.96$ $\left(s, 3 \mathrm{H}, \mathrm{CO}_{2} \mathrm{Me}\right), 3.80(s, 3 \mathrm{H}, \mathrm{OMe}), 3.40-3.33$ $\left(d, J=7.0 \mathrm{~Hz}, 2 \mathrm{H}, 16-\mathrm{H}_{2}\right), 3.22-3.16\left(m, 2 \mathrm{H}, 7-\mathrm{H}_{2}\right)$, $2.90-2.83\left(m, 2 \mathrm{H}, 8-\mathrm{H}_{2}\right), 2.10-2.02(m, 2 \mathrm{H}, 20-$
$\left.\mathrm{H}_{2}\right), 2.00-1.94\left(m, 2 \mathrm{H}, 19-\mathrm{H}_{2}\right), 1.79(d, J=1.3 \mathrm{~Hz}$, $3 \mathrm{H}, \mathrm{Me}), 1.65(d, J=1.4 \mathrm{~Hz}, 3 \mathrm{H}, \mathrm{Me}), 1.58$ $(d, J=1.3 \mathrm{~Hz}, 3 \mathrm{H}, \mathrm{Me}) \mathrm{ppm}$;

${ }^{13} \mathrm{C}$ NMR $\left(101 \mathrm{MHz}, \mathrm{CDCl}_{3}\right): \delta=172.1\left(\mathrm{CO}_{2} \mathrm{Me}\right)$, 161.9 (C-4), 161.3 (C-2), 144.0 (C-6), 142.0 (C-9), 135.0 (C-18), 131.1 (C-22), 128.4 (C-10), 125.9 (C-11), 124.5 (C-12), 122.9 (C-21), 122.2 (C-17), 115.3 (C-3), 105.9 (C-1), 105.2 (-C5), 55.4 (OMe), $52\left(\mathrm{CO}_{2} \mathrm{Me}\right), 39.8$ (C-19), $39.3(\mathrm{C}-8), 38.4(\mathrm{C}-7), 26.7$ (C-20), $25.7(\mathrm{Me}), 22.0$ (C-16), $17.6(\mathrm{Me}), 16.1$ (Me) ppm;

MS (ESI, MeOH): m/z = $391.2(92 \%,[\mathrm{M}+\mathrm{H}-$ $\left.\mathrm{MeOH}]^{+}\right), \quad 423\left(40 \%,[\mathrm{M}+\mathrm{H}]^{+}\right), 445.2(80 \%$, $\left.[\mathrm{M}+\mathrm{Na}]^{+}\right), \quad 461.2\left(40 \%,[\mathrm{M}+\mathrm{K}]^{+}\right), \quad 477.1 \quad(22 \%$, $[\mathrm{M}+\mathrm{Na}+\mathrm{MeOH}]^{+}$);

analysis calcd for $\mathrm{C}_{22} \mathrm{H}_{34} \mathrm{O}_{4}$ (422.57): C 76.74, $\mathrm{H}$ 8.11; found: C 76.50, H 8.32.

Data for 8: $\mathrm{R}_{\mathrm{F}}=0.46$ (n-hexane/ethyl acetate);

IR (ATR): $v=3086 v w, 3062 v w, 3026 v w, 2931 w$, $2859 w, 1725 s, 1602 s, 1584 m, 1496 w, 1453 m$, $1432 m, 1382 w, 1346 w, 1317 m, 1282 m, 1260 s$, $1225 m, 1195 s, 1157 v s, 1099 s, 1081 m, 1049 s$, $1030 m, 993 w, 949 w, 923 w, 881 w, 827 m, 817 m$, $788 w, 748 m, 700 s, 639 w, 609 w, 574 w, 561 w, 490 w$, $461 w \mathrm{~cm}^{-1}$;

UV/Vis $\left(\mathrm{CHCl}_{3}\right): \lambda_{\max }(\log \varepsilon)=228$ (4.52), 283 (3.71) $\mathrm{nm}$;

${ }^{1} \mathrm{H}$ NMR $\left(400 \mathrm{MHz}, \mathrm{CDCl}_{3}\right): \delta=7.32-7.24(m, 2 \mathrm{H}$, $11-\mathrm{H}), 7.24-7.15(m, 3 \mathrm{H}, 10-\mathrm{H}, 12-\mathrm{H}), 6.34(d, J=$ $2.3 \mathrm{~Hz}, 1 \mathrm{H}, 5-\mathrm{H}), 6.25(d, J=2.2 \mathrm{~Hz}, 1 \mathrm{H}, 3-\mathrm{H}), 5.43$ $(t, J=6.4 \mathrm{~Hz}, 1 \mathrm{H}, 17-\mathrm{H}), 5.09(t, J=6.8 \mathrm{~Hz}, 1 \mathrm{H}$, $21-\mathrm{H}), 4.55\left(d, J=6.3 \mathrm{~Hz}, 2 \mathrm{H}, 16-\mathrm{H}_{2}\right), 3.88(s, 3 \mathrm{H}$, $\left.\mathrm{CO}_{2} \mathrm{Me}\right), 3.74(s, 3 \mathrm{H}, \mathrm{OMe}), 2.93-2.78(m, 4 \mathrm{H}$, $\left.\left.8-\mathrm{H}_{2}, 7-\mathrm{H}_{2}\right), 2.14-2.01\left(m, 4 \mathrm{H}, 20-\mathrm{H}_{2}\right), 19-\mathrm{H}_{2}\right), 1.71$ $(s, 3 \mathrm{H}, \mathrm{Me}), 1.68(s, 3 \mathrm{H}, \mathrm{Me}), 1.60(s, 4 \mathrm{H}, \mathrm{Me}) \mathrm{ppm}$; ${ }^{13} \mathrm{C} \mathrm{NMR}\left(101 \mathrm{MHz}, \mathrm{CDCl}_{3}\right): \delta=168.8\left(\mathrm{CO}_{2} \mathrm{Me}\right)$, 161.2 (C-4), 157.5 (C-2), 141.8 (C-6), 141.6 (C-18), 140.6 (C-9), 131.8 (C-22), 128.4 (C-10), 128.3 (C-11), 126 (C-12), 123.8 (C-21), 119.6 (C-17), 116.8 (C-1), 106.1 (C-5), 98 (C-3), 66 (C-16), 55.3 (OMe), $52.0\left(\mathrm{CO}_{2} \mathrm{Me}\right), 39.5(\mathrm{C}-19), 39.3(\mathrm{C}-8), 38.4$ (C-7), 26.3 (C-20), $25.6(\mathrm{Me}), 17.7(\mathrm{Me}), 16.7(\mathrm{Me})$ ppm; MS (ESI, MeOH): m/z $581.4\left(70 \%,[\mathrm{M}+\mathrm{Na}]^{+}\right)$, $559.2\left(100 \%,[\mathrm{M}+\mathrm{H}]^{+}\right)$;

analysis calcd for $\mathrm{C}_{37} \mathrm{H}_{50} \mathrm{O}_{4}$ (558.80): C 79.53, H 9.02; found: C 79.37, H 9.25.

\section{Amorfrutin B, (E)-3-(3,7-dimethylocta-2,6-dien-1- yl)-2-hydroxy-4-methoxy-6-phenethyl benzoic acid (2) \\ From 7: A solution of $7(100 \mathrm{mg}, 0.24 \mathrm{mmol})$ in $\mathrm{MeOH}(5 \mathrm{~mL})$ was added to a $40^{\circ} \mathrm{C}$ solution of $\mathrm{KOH}$ (84 mg, $1.5 \mathrm{mmol})$ in $\mathrm{MeOH} / \mathrm{H}_{2} \mathrm{O}(7: 1,40 \mathrm{~mL})$, and the mixture was heated under reflux for $10 \mathrm{~h}$. Usual workup followed by chromatography (silica gel, $n$ - hexane/ethyl acetate, 3:1) gave $2(81 \mathrm{mg}, 84 \%)$ as a colorless solid.}

From 8: Following the procedure given for the synthesis of 4 (from 6) from 8 compound 2 (72\%) was obtained. An analytical sample showed: m.p. $74-76^{\circ} \mathrm{C}$ 
(lit: $\left.{ }^{50} 80.2-83.1^{\circ} \mathrm{C}\right) ; \quad \mathrm{R}_{\mathrm{F}}=0.23$ (n-hexane/ethyl acetate, $3: 1)$;

IR (ATR): $v=3064 w, 3028 w, 2961 m, 2925 b, 2855 w$, $2671 w, 2592 w, 2537 w, 1633 s, 1607 s, 1571 m, 1496 m$, $1453 \mathrm{~m}, 1430 \mathrm{~m}, 1401 \mathrm{~m}, 1380 \mathrm{~m}, 1348 \mathrm{w}, 1266 \mathrm{vs}$, $1221 \mathrm{~s}, 1188 \mathrm{~m}, 1170 \mathrm{~m}, 1149 \mathrm{~m}, 1113 \mathrm{~s}, 1077 \mathrm{~m}$, $1046 w, 1030 w, 1003 w, 983 w, 923 w, 902 m, 858 w$, $837 m, 819 m, 804 m, 773 m, 749 s, 734 m, 696 s, 679 m$, $663 w, 608 m, 562 w, 533 w, 494 m \mathrm{~cm}^{-1}$; UV/Vis $\left(\mathrm{CHCl}_{3}\right): \lambda_{\max }(\log \varepsilon)=229$ (4.07), 277 (3.76), 313 (3.26) $\mathrm{nm}$;

${ }^{1} \mathrm{H}$ NMR $\left(400 \mathrm{MHz}, \mathrm{CDCl}_{3}\right): \delta=11.56(s, 2 \mathrm{H}, \mathrm{COOH}$ $+\mathrm{OH}), 7.33-7.27(m, 2 \mathrm{H}, 11-\mathrm{H}), 7.23-7.18(m$, $3 \mathrm{H}, 10-\mathrm{H}, 12-\mathrm{H}), 6.22(s, 1 \mathrm{H}, 5-\mathrm{H}), 5.21(t, J=5.9$ $\mathrm{Hz}, 1 \mathrm{H}, 16-\mathrm{H}), 5.08(t, J=6.9 \mathrm{~Hz}, 1 \mathrm{H}, 20-\mathrm{H}), 3.79$ $(s, 3 \mathrm{H}, \mathrm{OMe}), 3.36\left(d, J=7.0 \mathrm{~Hz}, 2 \mathrm{H}, 15-\mathrm{H}_{2}\right), 3.32-$ $3.23\left(m, 2 \mathrm{H}, 7-\mathrm{H}_{2}\right), 2.98-2.89\left(m, 2 \mathrm{H}, 8-\mathrm{H}_{2}\right), 2.11-$ $1.93\left(m, 4 \mathrm{H}, 19-\mathrm{H}_{2}, 18-\mathrm{H}_{2}\right), \quad 1.81-1.76(m, 3 \mathrm{H}, \mathrm{Me})$, $1.68-1.62(m, 3 \mathrm{H}, \mathrm{Me}), 1.60-1.56(m, 3 \mathrm{H}, \mathrm{Me})$ ppm;

${ }^{13} \mathrm{C}$ NMR $\left(101 \mathrm{MHz}, \mathrm{CDCl}_{3}\right): \delta=175.6(\mathrm{COOH})$, 162.9 (C-4), 162.2 (C-2), 145.8 (C-6), 141.9 (C-9), 135.2 (C-17), 131.1 (C-21), 128.5 (C-10), 128.4 (C-11), 125.9 (C-12), 124.5 (C-20), 122.1 (C-16), 115.5 (C-3), 106.5 (C-5), 103.7 (C-1), 55.5 (OMe), 39.8 (C-18), 39.2 (C-8), 38.1 (C-7), 26.8 (C-19), 25.7 (Me), 21.9 (C-15), 17.7 (Me), 16.1 (Me) ppm; MS (ESI, MeOH): $\mathrm{m} / \mathrm{z}=391.2(100 \%,[\mathrm{M}+\mathrm{H}-$ $\left.\left.\mathrm{H}_{2} \mathrm{O}\right]^{+}\right), 409\left(46 \%,[\mathrm{M}+\mathrm{H}]^{+}\right), 431.2\left(98 \%,[\mathrm{M}+\mathrm{Na}]^{+}\right)$ and $\left.\mathrm{m} / \mathrm{z}=363.2(22 \% \text {, [M-H-CO }]^{-}\right), 407.2(100 \%$, $\left.[\mathrm{M}-\mathrm{H}]^{-}\right)$;

analysis calcd for $\mathrm{C}_{26} \mathrm{H}_{32} \mathrm{O}_{4}$ (408.54): C 76.44, H 7.90; found: C 76.20, H 8.03.

\section{3,6-Bis(diethylamino)-9-[2-(1- piperazinylcarbonyl)phenyl]-xanthylium chloride (9)}

This compound was prepared as previously reported from rhodamine $\mathrm{B}$, oxalyl chloride and piperazine ${ }^{38}$ in $67 \%$ yield as a dark purple solid; m.p. $>350^{\circ} \mathrm{C}$; $\mathrm{R}_{\mathrm{F}}=0.15$ (chloroform/methanol, 8:2); $\lambda_{\max }(\log \varepsilon)=$ 260 (0.23), 354 (0.06), $561(0.82) \mathrm{nm}$;

IR (ATR) $v=3401 b r, 1589 m, 1529 w, 1411 s, 1328 s$, $1275 \mathrm{~s}, 1246 m, 1180 \mathrm{~s}, 1132 \mathrm{~m}, 1074 \mathrm{~m}, 1011 \mathrm{w}, 977 \mathrm{~m}$ $922 m, 820 m, 683 m$;

${ }^{1} \mathrm{H}$ NMR (500 MHz, $\left.\mathrm{CD}_{3} \mathrm{OD}\right): \delta=7.79-7.74(m, 3 \mathrm{H}$, $3-\mathrm{H}+4-\mathrm{H}+5-\mathrm{H}), 7.52(m, 1 \mathrm{H}, 6-\mathrm{H}), 7.28-7.25$ $(d, 1 \mathrm{H}, 10-\mathrm{H}), 7.10-7.09(m, 1 \mathrm{H}, 11-\mathrm{H}), 6.98-6.97$ $(d, 1 \mathrm{H}, 13-\mathrm{H}), 3.72-3.59\left(m, 6 \mathrm{H}, 15-\mathrm{H}_{\mathrm{a}}+15-\mathrm{H}_{\mathrm{b}}+\right.$ $\left.17-\mathrm{H}_{\mathrm{a}}+17-\mathrm{H}_{\mathrm{b}}+20-\mathrm{H}_{\mathrm{a}}+20-\mathrm{H}_{\mathrm{b}}\right), 3.08-3.05(t, 4 \mathrm{H}$, $\left.18-\mathrm{H}_{\mathrm{a}}+18-\mathrm{H}_{\mathrm{b}}+19-\mathrm{H}_{\mathrm{a}}+19-\mathrm{Hb}\right), 1.33-1.30(t, 3 \mathrm{H}$, $\left.16-\mathrm{H}_{\mathrm{a}}+16-\mathrm{H}_{\mathrm{b}}+16-\mathrm{H}_{\mathrm{c}}\right) \mathrm{ppm}$;

${ }^{13} \mathrm{C}$ NMR (126 MHz, CD $\left.{ }_{3} \mathrm{OD}\right): \delta=169.53(\mathrm{C}-1)$, 159.2 (C-8), 157.3 (C-12), 156.7 (C-14), 135.7 (C-7), 133.0 (C-10), 132.3 (C-2), 131.8 (C-6), 131.5 (C-5), 131.4 (C-4), 128.9 (C-3), 115.4 (C-11), 114.8 (C-9), 97.4 (C-13), 46.9 (C-15), 46.8 (C-17 + C-20), 44.5 (C-18 + C-19), 12.8 (C16) ppm;

MS (ESI, MeOH): $m / z=256.4\left(24 \%,[\mathrm{M}+\mathrm{H}]^{2+}\right)$, $511.3\left(100 \%,[\mathrm{M}]^{+}\right)$; analysis calcd for $\mathrm{C}_{32} \mathrm{H}_{39} \mathrm{ClN}_{4} \mathrm{O}_{2}$ (547.14): $\mathrm{C} 70.25, \mathrm{H}$ 7.18, N 10.24; found: C 70.07, H 7.30, N 10.01.

\section{$N$-(6-(Diethylamino)-9-(2-(4-(2-hydroxy-4- methoxy-6-phenethylbenzoyl)piperazine-1- carbonyl)phenyl)-3H-xanthen-3-ylidene)- $\mathrm{N}$ - ethylethanaminium chloride (10)}

To a solution of $\mathbf{3}(181 \mathrm{mg}, 0.66 \mathrm{mmol})$ in dry DCM (30 mL), EDC HCl (153 mg, $0.79 \mathrm{mmol})$, HOBt $(120 \mathrm{mg}, 0.79 \mathrm{mmol})$ and $9(361 \mathrm{mg}, 0.66 \mathrm{mg})$ were added; the mixture was stirred for $12 \mathrm{~h}$. Usual workup followed by chromatography (silica gel, $\left.\mathrm{CHCl}_{3} / \mathrm{MeOH}, 1 \% \rightarrow 15 \%\right)$ gave $\mathbf{1 0}(392 \mathrm{mg}, 74 \%)$ as a pink amorphous solid; $\mathrm{R}_{\mathrm{F}}=0.2\left(\mathrm{CHCl}_{3} / \mathrm{MeOH}\right.$, 9:1);

IR (ATR): $v=3061 w, 2974 w, 2932 w, 2869 w$, $1629 \mathrm{~m}, 1584 \mathrm{vs}, 1558 \mathrm{~m}, 1528 \mathrm{~m}, 1506 \mathrm{~m}, 1481 \mathrm{~m}$, $1464 m, 1456 m, 1410 s, 1393 s, 1333 v s, 1271 s, 1245 s$, $1196 m, 1178 v s, 1158 s, 1130 s, 1072 s, 1048 m, 1000 s$, $977 m, 921 m, 868 m, 821 s, 787 m, 754 m, 702 m, 682 s$, $664 m, 642 m, 620 m, 581 m, 546 m, 523 m, 497 m \mathrm{~cm}^{-1}$; UV/Vis $\left(\mathrm{CHCl}_{3}\right): \lambda_{\max }(\log \varepsilon)=229$ (4.57), 261 (4.48), 357 (3.85), 563 (5.01) nm;

${ }^{1} \mathrm{H}$ NMR $\left(400 \mathrm{MHz}, \mathrm{CDCl}_{3}\right): \delta=10.08(\mathrm{~s}, 1 \mathrm{H}, \mathrm{OH})$, $7.66(\mathrm{~s}, 1 \mathrm{H}, 23-\mathrm{H}), 7.60(\mathrm{dd}, J=5.9,2.9 \mathrm{~Hz}, 2 \mathrm{H}$, 21-H, 24-H), 7.43 (s, 1H, 22-H), $7.33-7.26$ (m, 2H, 11-H), $7.25-7.20(\mathrm{~m}, 2 \mathrm{H}, 28-\mathrm{H}, 37-\mathrm{H}), 7.20-7.12$ (m, 3H, 10-H, 12-H), 6.78 (dd, $J=9.4,2.4 \mathrm{~Hz}, 2 \mathrm{H}$, 29-H, 36-H), 6.70 (d, $J=2.4 \mathrm{~Hz}, 2 \mathrm{H}, 31-\mathrm{H}, 34-\mathrm{H})$, 6.66 (s, 1H, 3-H), 6.27 (d, J=2.3 Hz, 1H, 5-H), 4.37 $\left(\mathrm{s}, 2 \mathrm{H}, 15 \mathrm{a}-\mathrm{H}_{2}, 17 \mathrm{a}-\mathrm{H}_{2}\right), 3.94\left(\mathrm{~s}, 2 \mathrm{H}, 16 \mathrm{a}-\mathrm{H}_{2}, 18 \mathrm{a}-\right.$ $\left.\mathrm{H}_{2}\right), 3.73(\mathrm{~s}, 3 \mathrm{H}, \mathrm{OMe}), 3.67-3.50\left(\mathrm{~m}, 8 \mathrm{H}, 39-\mathrm{H}_{2}\right.$, $\left.41-\mathrm{H}_{2}, 43-\mathrm{H}_{2}, 45-\mathrm{H}_{2}\right), 3.06-2.96\left(\mathrm{~m}, 2 \mathrm{H}, 7-\mathrm{H}_{2}\right), 2.89$ (s, 4H, 15b- $\left.\mathrm{H}_{2}, 16 \mathrm{~b}-\mathrm{H}_{2}, 17 \mathrm{~b}-\mathrm{H}_{2}, 18 \mathrm{~b}-\mathrm{H}_{2}\right), 1.32$ (dt, $J=$ 17.3, 7.0 Hz, 12H, 4 x Me) ppm;

${ }^{13} \mathrm{C}$ NMR (101 MHz, $\left.\mathrm{CDCl}_{3}\right): \delta=173.2(\mathrm{CO}), 168.3$ (CO), 167.4 (C-4), 161 (C-2), 157.8 (C-27, C-38), 157.5 (C-25), 155.3 (C-30, C-35), 142 (C-6), 140.8 (C-9), 135.4 (C-20), 132.3 (C-28, C-37), 131.4 (C-21), 131.2 (C-26), 130.3 (C-10), 129.8 (C-22), 128.6 (C-23), 128.3 (C-11), 127.4 (C-24), 125.8 (C-12), 115.2(C-1), 113.9 (C-32), 113.5(C-33), 113.3 (C-29, C-36), 107.6 (C-5), 99.1 (C-3), 96.1 (C-31, C-34), 55.3 (OMe), 45.9 (C-39, C-41, C-43, C-45), 41.8 (C-7), 37.5 (C-8), 35.4 (C-15, C-16, C-17, C-18), 12.6 (4 x Me) ppm;

MS (ESI, MeOH): $\mathrm{m} / \mathrm{z}=765.3\left(100 \%,[\mathrm{M}-\mathrm{Cl}]^{+}\right.$; analysis calcd for $\mathrm{C}_{48} \mathrm{H}_{53} \mathrm{~N}_{4} \mathrm{O}_{5} \mathrm{Cl}(801.43)$ : $\mathrm{C} 71.94, \mathrm{H}$ 6.67, N 6.99; found: C 71.77, H 6.81, N 6.67.

\section{References}

1- D.J. Newman, Developing natural product drugs: Supply problems and how they have been overcome, Pharmacol. Therapeut., 2016, 162, 1-9.

2- D.J. Newman, G.M. Cragg, Natural Products as Sources of New Drugs from 1981 to 2014, J. Nat. Prod., 2016, 79, 629-661.

3- D.J. Newman, G.M. Cragg, Natural Product Scaffolds of Value in Medicinal Chemistry, RSC Drug Discov., 2016, 50, 348-378. 
4- H. von Bingen, Komplette Werkausgabe, Abtei St. Hildegard, Eibingen, Germany, 2017.

5- M. Treben, Health through God's pharmacy, Ennsthaler Verlag, Steyr, Austria, 2007.

6- https://www.who.int/new-room/factsheets/detail/the-top-10-causes-of-death;last accessed 2020-11-17.

7- D. Yach, D. Stuckler, K.D. Brownell, Epidemiologic and economic consequences of the global epidemics of obesity and diabetes, Nat. Med., 2006, 12, 62-66.

8- S. Sauer, Amorfrutins: A Promising Class of Natural Products that Are Beneficial to Health, ChemBioChem., 2014, 15, 1231-1238.

9- D. Raederstorff, Novel nutraceutical, and pharmaceutical compositions comprising methoxylated aromatic compounds for the treatment, co-treatment or prevention of inflammatory disorders, 2007, WO2007093387A2.

10-J.L. Rios, F. Francini, G.R. Schinella, Natural Products for the Treatment of Type 2 Diabetes Mellitus, Planta Med., 2015, 81, 975-994.

11-L.A. Mitscher, Y.H. Park, A. Al-Shamma, P.B. Hudson, T. Haas, Amorfrutin A and B, bibenzyl antimicrobial agents from Amorpha fruticosa, Phytochemistry, 1981, 20, 781-785.

12-R. Muharini, A. Diaz, W. Ebrahim, A. Mandi, T. Kurtan, N. Rehberg, R. Kalscheuer, R. Hartmann, R.S. Orfali, W. Lin, Z. Liu, P. Proksch, Antibacterial and Cytotoxic Phenolic Metabolites from the Fruits of Amorpha fruticosa, J. Nat. Prod., 2017, 80, 169-180.

13-C. Weidner, J.C. de Groot, A. Prasad, A. Freiwald, C. Quedenau, M. Kliem, A. Witzke, V. Kodelja, C.T. Han, S. Giegold, M. Baumann, B. Klebl, K. Siems, L. Muller-Kuhrt, A. Schurmann, R. Schuler, A.F.H. Pfeiffer, F.C. Schroeder, K. Bussow, S. Sauer, Amorfrutins are potent antidiabetic dietary natural products, P. Natl. Acad. Sci., 2012, 109, 7257-7262.

14-L. Fuhr, M. Rousseau, A. Plauth, F.C. Schroeder, S. Sauer, Amorfrutins Are Natural PPAR gamma Agonists with Potent Anti-inflammatory Properties, J. Nat. Prod., 2015, 78, 1160-1164.

15-C. Chen, Y. Wu, L.L. Du, Qualitative and quantitative analysis of amorfrutins, novel antidiabetic dietary natural products, by HPLC, Pharm. Biol., 2016, 54, 488-493.

16-C. Weidner, M. Rousseau, R.J. Micikas, C. Fischer, A. Plauth, S.J. Wowro, K. Siems, G. Hetterling, M. Kliem, F.C. Schroeder, S. Sauer, Amorfrutin C Induces Apoptosis and Inhibits Proliferation in Colon Cancer Cells through Targeting Mitochondria, J. Nat. Prod., 2016, 79, 2-12.

17-J.C. de Groot, C. Weidner, J. Krausze, K. Kawamoto, F.C. Schroeder, S. Sauer, K. Buessow, Structural Characterization of Amorfrutins Bound to the Peroxisome Proliferator-Activated Receptor $\gamma$, J. Med. Chem., 2013, 56, 1535-1543.
18-C. Weidner, S.J. Wowro, A. Freiwald, K. Kawamoto, A. Witzke, M. Kliem, K. Siems, L. Mueller-Kuhrt, F.C. Schroeder, S. Sauer, Amorfrutin B is an efficient natural peroxisome proliferator-activated receptor gamma (PPAR $\gamma$ ) agonist with potent glucose-lowering properties, Diabetologia, 2013, 56, 1802-1812.

19-L. Wang, B. Waltenberger, E.M. PferschyWenzig, M. Blunder, X. Liu, C. Malainer, T. Blazevic, S. Schwaiger, J.M. Rollinger, E.H. Heiss, D. Schuster, B. Kopp, R. Bauer, H. Stuppner, V.M. Dirsch, A.G. Atanasov, Natural product agonists of peroxisome proliferator-activated receptor-gamma (PPAR $\gamma)$ : a review, Biochem. Pharmacol., 2014, 92, 73-89.

20-S. Garcia-Vallve, L. Guasch, S. TomasHernandez, J.M. del Bas, V. Ollendorff, L. Arola, G. Pujadas, M. Mulero, Peroxisome ProliferatorActivated Receptor $\gamma$ (PPAR $\gamma$ ) and Ligand Choreography: Newcomers Take the Stage, J. Med. Chem., 2015, 58, 5381-5394.

21-A. Lavecchia, C. Di Giovanni, Amorfrutins are efficient modulators of peroxisome proliferatoractivated receptor-gamma (PPAR $\gamma)$ with potent antidiabetic and anticancer properties: a patent evaluation of WO2014177593 A1, Expert Opin. Ther. Pat., 2015, 25, 1341-1347.

22-S. Yousefnia, S. Momenzadeh, F. Seyed Forootan, K. Ghaedi, M.H. Nasr Esfahani, The influence of peroxisome proliferator-activated receptor $\gamma(\operatorname{PPAR} \gamma)$ ligands on cancer cell tumorigenicity, Gene, 2018, 649, 14-22.

23-D. Raederstorff, J. Schwager, K. Wertz, Composition comprising rosehip for treatment of the inflammatory disorder, $\mathbf{2 0 0 8}$, WO2008006589A2.

24-S. Liu, M. Su, S.J. Song, J. Hong, H.Y. Chung, J.H. Jung, An Anti-Inflammatory PPAR- $\gamma$ Agonist from the Jellyfish-Derived Fungus Penicillium chrysogenum J08NF-4, J. Nat. Prod., 2018, 81, 356-363.

25-P. Lefebvre, B. Staels, Naturally improving insulin resistance with amorfrutins, Proc. Natl. Acad. Sci., 2012, 109, 7136-7137.

26-D. Raederstorff, J. Schwager, G. Schueler, Nutraceutical and pharmaceutical compositions and use thereof for the treatment, co-treatment or prevention of inflammatory disorders, 2012, WO2007093387A1.

27-C. Chen, Y. Xue, Q.M. Li, Y. Wu, J. Liang, L.S. Qing, Neutral Loss Scan - Based Strategy for Integrated Identification of Amorfrutin Derivatives, New Peroxisome ProliferatorActivated Receptor Gamma Agonists, from Amorpha Fruticosa by UPLC-QqQ-MS/M.S. and UPLCQ-TOF-MS, J. Am. Soc. Mass Spectrom., 2018, 29, 685-693.

28-S. Laclef, K. Anderson, A.J.P. White, A.G.M. Barrett, Total synthesis of amorfrutin A via a palladium-catalyzed migratory prenylationaromatization sequence, Tetrahedron Lett., 2012, 53, 225-227. 
29-C. Weidner, J.C. de Groot, A. Prasad,

A. Freiwald, C. Quedenau, M. Kliem, A. Witzke, V. Kodelja, C.T. Han, S. Giegold, M. Baumann, B. Klebl, K. Siems, L. Muller-Kuhrt,

A. Schurmann, R. Schuler, A.F.H. Pfeiffer, F.C. Schroeder, K. Bussow, S. Sauer, Amorfrutins are potent antidiabetic dietary natural products, Proc. Natl. Acad. Sci., 2012, 109, 7257-7262.

30-Y.Y. Song, H.G. He, Y. Li, Y. Deng, A facile total synthesis of amorfrutin A, Tetrahedron Lett., 2013, 54, 2658-2660.

31-I.S. Aidhen, R. Mukkamala, C. Weidner, S. Sauer, A Common Building Block for the Syntheses of Amorfrutin and Cajaninstilbene Acid Libraries Toward Efficient Binding with Peroxisome Proliferator-Activated Receptors, Org. Lett., 2015, 17, 194-197.

32-X.Y. Ji, J.H. Chen, G.H. Zheng, M.H. Huang, L. Zhang, H. Yi, J. Jin, J.-D. Jiang, Z.G. Peng, Z.R. Li, Design, and Synthesis of Cajanine Analogues against Hepatitis $\mathrm{C}$ Virus through Down-Regulating Host Chondroitin Sulfate NAcetylgalactosaminyltransferase 1, J. Med. Chem., 2016, 59, 10268-10284.

33-T. Fujita, S. Kuwahara, Y. Ogura, Unified total synthesis of amorfrutins A and C via the Claisen rearrangement, Biosci. Biotechnol. Biochem., 2019, 83, 1635-1641.

34-B. Weber, B. Brandes, D. Powroznik, R. Kluge, R. Csuk, An efficient and robust synthesis of amorfrutin A, Tetrahedron Lett., 2019, 60, 1379-1381.

35-T. Fujita, S. Kuwahara, Y. Ogura, Synthesis of amorfrutins B and D from amorfrutin A ethyl ester, Tetrahedron Lett., 2020, 61, 151477.

36-G.S. Grandhi, J. Selvakumar, S. Dana, M. Baidya, Directed C-H Bond Functionalization: A Unified Approach to Formal Syntheses of Amorfrutin A, Cajaninstilbene Acid, Hydrangenol, and Macrophyllol, J. Org. Chem., 2018, 83, 12327-12333.

37-E.L. Ghisalberti, P.R. Jefferies, D. McAdam, Isoprenylated resorcinol derivatives from Glycyrrhiza acanthocarpa, Phytochemistry, 1981, 20, 1959-1961.

38-M. Kozubek, I. Serbian, S. Hoenke, O. Kraft, R. Csuk, Synthesis and cytotoxic evaluation of hydroxycinnamic acid rhodamine B conjugates, Results Chem., 2020, 2, 100057.

39-S. Sommerwerk, L. Heller, C. Kerzig, A.E. Kramell, R. Csuk, Rhodamine B conjugates of triterpenoic acids are cytotoxic mitocans even at nanomolar concentrations, Eur. J. Med. Chem., 2017, 127, 1-9.
40-M. Kahnt, J. Wiemann, L. Fischer, S. Sommerwerk, R. Csuk, Transformation of asiatic acid into a mitocanic, bimodal-acting rhodamine B conjugate of nanomolar cytotoxicity, Eur. J. Med. Chem., 2018, 159, 143-148.

41-J. Wiemann, L. Fischer, J. Kessler, D. Ströhl, R. Csuk, Ugi multicomponent-reaction: Syntheses of cytotoxic dehydroabietylamine derivatives, Bioorg Chem., 2018, 81, 567-576.

42-R.K. Wolfram, L. Fischer, R. Kluge, D. Ströhl, A. Al-Harrasi, R. Csuk, Homopiperazinerhodamine $\mathrm{B}$ adducts of triterpenoic acids are strong mitocans, Eur. J. Med. Chem., 2018, 155, 869-879.

43-R.K. Wolfram, L. Heller, R. Csuk, Targeting mitochondria: Esters of rhodamine $\mathrm{B}$ with triterpenoids are mitocanic triggers of apoptosis, Eur. J. Med. Chem., 2018, 152, 21-30.

44-R. Csuk, H.P. Deigner, The potential of click reactions for the synthesis of bioactive triterpenes, Bioorg. Med. Chem. Lett., 2019, 29, 949-958.

45-B. Brandes, S. Hoenke, L. Fischer, R. Csuk, Design, synthesis, and cytotoxicity of BODIPY FL labelled triterpenoids, Eur. J. Med. Chem., 2020, 185, 111858.

46-S. Friedrich, I. Serbian, S. Hoenke, R.K. Wolfram, R. Csuk, Synthesis and cytotoxic evaluation of malachite green derived oleanolic and ursolic acid piperazineamides, Med. Chem. Res., 2020, 29, 926-933.

47-B. Del Secco, G. Malachin, L. Milli, N. Zanna, E. Papini, A. Cornia, R. Tavano, C. Tomasini, Form matters: Stable helical foldamers preferentially target human monocytes and granulocytes, ChemMedChem, 2017, 12, 337-345.

48-A.N. Preston, J.D. Farr, B.K. O'Neill, K.K. Thompson, S.E. Tsirka, S.T. Laughlin, Visualizing the Brain's Astrocytes with Diverse Chemical Scaffolds, ACS Chem. Biol., 2018, 13, 1493-1498.

49-I. Serbian, S. Hoenke, R. Csuk, Synthesis of some steroidal mitocans of nanomolar cytotoxicity acting by apoptosis, Eur. J. Med. Chem., 2020, 199, 112425.

50-X.-J. Xu, T. Zeng, Z.X. Huang, X.F. Xu, J. Lin, W.M. Chen, Synthesis, and Biological Evaluation of Cajaninstilbene Acid and Amorfrutins A and B as Inhibitors of the Pseudomonas aeruginosa Quorum Sensing System, J. Nat. Prod., 2018, 81, 2621-2629. 